\title{
ON CHARACTERIZATIONS OF WEIGHTED COMPOSITION OPEARTORS ON NON-LOCALLY CONVEX WEIGHTED SPACES OF CONTINUOUS FUNCTIONS
}

\author{
S. D. SHARMA, KAMALJEET KOUR AND BHOPINDER SINGH
}

\begin{abstract}
For a system $V$ of weights on a completely regular Hausdorff space $X$ and a Hausdorff topological vector space $E$, let $C V_{b}(X, E)$ and $C V_{0}(X, E)$ respectively denote the weighted spaces of continuouse $E$-valued functions $f$ on $X$ for which $(v f)(X)$ is bounded in $E$ and $v f$ vanishes at infinity on $X$ all $v \in V$. On $C V_{b}(X, E)\left(C V_{0}(X, E)\right)$, consider the weighted topology, which is Hausdorff, linear and has a base of neighbourhoods of 0 consising of all sets of the form: $N(v, G)=\{f:(v f)(X) \subseteq G\}$, where $v \in V$ and $G$ is a neighbourhood of 0 in $E$. In this paper, we characterize weighted composition operators on weighted spaces for the case when $V$ consists of those weights which are bounded and vanishing at infinity on $X$. These results, in turn, improve and extend some of the recent works of Singh and Singh [10, 12] and Manhas [6] to a non-locally convex setting as well as that of Singh and Manhas [14] and Khan and Thaheem [4] to a larger class of operators.
\end{abstract}

\section{Introduction}

The contents of this paper are in relation with the theory of weighted composition operators on weighted spaces which are studied by Jamison and Rajagopalan [1], Singh and Summers [17], Singh and Manhas [14], Singh and one of the authors in [10, 12], Khan and Thaheem [3, 4], Manhas [6], and two of the authors in [8, 9]. In [17], Singh and Summers have made a detailed study of composition operators on locally convex weighted spaces where as multiplication operators on such spaces have been studied by Singh and Manhas [14] and their results have been generalized by Singh and Singh [10] to a larger class of operators, known as weighted composition operators. Khan and Thaheem, in a very recent work [4], have extended the work of [14] to a non-locally convex setting and their work have been further extended by Singh and Kour [8]. This paper is a continuation of earlier paper [12] in which a characterization of weighted composition operators on locally convex weighted space $C V_{b}(X, E)$ is presented and also it is a continuation to earlier paper [9] where we have studied weighted composition

Received May 28, 1998; revised October 20, 1999.

1991 Mathematics Subject Classification. 47B38, 46E40, 46A16.

Key words and phrases. System of weights, weighted topology, shrinkable neighbourhood, topological vector space, vector-valued continuous function, weighted composition operator. 
operators $W_{\pi, T}\left(W_{\theta, T}\right)$ on non-locally convex weighted spaces $C V_{b}(X, E)$ and $C V_{0}(X, E)$ induced by $\pi: X \rightarrow E(\theta: X \rightarrow \mathbb{C})$ and $T: X \rightarrow X$.

The purpose of this paper is to characterize those weighted composition operators on non-locally convex weighted spaces which are induced by mappings $\pi: X \rightarrow B(E)$ and $T: X \rightarrow X$. These results improve and extend, in particular, some of the results contained in $[4,6,9,10,12,14]$.

\section{Preliminaries}

Throughout this paper we shall assume, unless stated otherwise, that $X$ is a completely regular Hausdorff space and $E$ is a non-trivial Hausdorff topological vector space over $\mathbb{K} \in\{\mathbb{R}, \mathbb{C}\}$. Then by $C(X, E)$ we denote the vector space of all continuous functions from $X$ into $E$. A function $f: X \rightarrow E$ is said to vanish at infinity if for each neighbourhood $N$ of origin in $E$ there exists a compact subset $K$ of $X$ such that $f(x) \in N$ for all $x$ in $X \backslash K$, the complement of the set $K$ in $X$. A subset $B$ of $E$ is said to be bounded if for every neighbourhood $N$ of 0 there exists $\varepsilon>0$ such that $B \subseteq \varepsilon N$. Then we define

$$
\begin{aligned}
& C_{0}(X, E)=\{f \in C(X, E): f \text { vanishes at infinity on } X\}, \text { and } \\
& C_{b}(X, E)=\{f \in C(X, E): f(X) \text { is bounded in } E\}, \text { where } f(X)=\{f(x): x \in X\} .
\end{aligned}
$$

Clearly $C_{0}(X, E) \subset C_{b}(X, E)$. When $E=\mathbb{K}$ with the usual topology, these spaces are respectively denoted by $C(X), C_{0}(X)$ and $C_{b}(X)$. In case $X=\mathbb{N}$, the set of all natural numbers with the discrete topology, $C_{b}(\mathbb{N})=l^{\infty}$, the Banach algebra of all bounded sequences in $\mathbb{K}$, and $C_{0}(\mathbb{N})=c_{0}$, the Banach space of null sequences in $\mathbb{K}$. A real-valued function $f$ on $X$ is called upper-semicontinuous if the set $\{x \in X: f(x)<a\}$ is open for all $a$ in $\mathbb{R}$. By a weight we mean a non negative upper-semicontinuous function on $X$. Let $V$ denote a family of weights on $X$. Then we say that $V>0$ if for every $x \in X$ there is some $v_{x} \in V$ such that $v_{x}(x)>0$; and that $V$ is direct upward (or a Nachbin family) if for every pair $u, v \in V$ and every $a>0$ there exists a $w \in V$ such that $a u(x) \leq w(x)$ and $a v(x) \leq w(x)$ for all $x$ in $X$. Since there is no loss of generality, we hereafter assume that the sets of weights are directed upward. Now by a system of weights we mean a set $V$ of weights on $X$ which additionally satisfies that $V>0$.

Let us now consider the following vector spaces (over $\mathbb{K}$ ) of continuous functions from $X$ into $E$ for a given system $V$ of weights on $X$ :

$$
\begin{aligned}
& C V_{0}(X, E)=\{f \in C(X, E): v f \text { vanishes at infinity on } X \text { for all } v \in V\} \text { and } \\
& C V_{b}(X, E)=\{f \in C(X, E):(v f)(X) \text { is bounded in } E \text { for all } v \in V\}
\end{aligned}
$$

where $(v f)(X)=\{v(x) f(x): x \in X\}$.

It is clear that $C V_{0}(X, E) \subset C V_{b}(X, E)$. On $C V_{b}(X, E)$, consider the weighted topology $w_{V}$, which is Hausdorff, linear and has a base of neighbourhoods of 0 consisting of all sets of the form:

$N(v, G)=\{f:(v f)(X) \subseteq G\}$, where $v \in V$ and $G$ is a neighbourhood of 0 in $E$. 
The space $C V_{b}(X, E)$, equipped with $w_{V}$ is called a weighted space. The space $C V_{0}(X, E)$, being a subspace of $C V_{b}(X, E)$, is equipped with the topology induced by $C V_{b}(X, E)$.

The following are some instances of weighted spaces:

(i) If $V$ is the set of all non-negative constant functions on $X$, then $C V_{b}(X, E)=$ $C_{b}(X, E)$ and $C V_{0}(X, E)=C_{0}(X, E)$. The topology $w_{V}$ in this case is the topology of uniform convergence.

(ii) If $V=\left\{a \chi_{K}: a \geq 0\right.$ and $K \subset X, K$ compact $\}$, where $\chi_{K}$ denotes the characteristic function of $K$, then $C V_{b}(X, E)=C V_{0}(X, E)=C(X, E)$ and $w_{V}$ is the compactopen topology.

(iii) If $V$ is the system of all non-negative weights which vanish at infinity on $X$, then $C V_{b}(X, E)=C V_{0}(X, E)=C_{b}(X, E)$ and $w_{V}$ in this case is the substrict topology.

For more details on such weighted spaces, we refer to Nachbin [7], Singh and Summers [17], Khan [2] and Khan and Thaheen [3].

Let $B(E)$ denote the vector space of all continuous linear mappings from $E$ into itself, endowed with the linear topology which has a base of neighbourhoods of 0 consisting of all sets of the form:

$U(B, G)=\{A \in B(E): A(B) \subseteq G\}$, where $B$ is bounded (or a finite) subset of $E$ and $G$ is a neighbourhood of 0 in $E$. By $B_{u}(E)$ (respectively, $B_{s}(E)$ ), we denote the space $B(E)$ when it is equipped with the uniform (respectively, strong) operator topology, that is, the topology of uniform (pointwise) convergence on bounded (finite) subsets of $E$.

Let $L(X, E)$ denote a vector space of functions from $X$ into $E$. If $\pi: X \rightarrow B(E)$ and $T: X \rightarrow X$ are mappings such that $\pi . f o T \in L(X, E)$ for every $f \in L(X, E)$, then the correspondence $f \rightarrow \pi . f o T$ is a linear transformation from $L(X, E)$ into itself and we denote it by $W_{\pi, T}$ (here the multiplication of $\pi$ and the composite function foT is defined as $\pi . f o T(x)=\pi(x)(f(T(x))$ for all $x \in X)$. In case $L(X, E)$ is a topological vector space and $W_{\pi, T}$ is continuous, it is called a weighted composition operator (in short, written as WCO) on $L(X, E)$ induced by the pair $(\pi, T)$.

In case $T$ is the identity map on $X$, the corresponding operator $W_{\pi, T}$ is called the multiplication operator and is denoted by $M_{\pi}$. On the other hand, when $\pi(x)=I$, the identity operator on $E$, for all $x \in X$, the corresponding WCO is called the composition operator and is denoted by $C_{T}$. For a detailed account of these operators on spaces of continuous functions, we refer to the monograph [16] of Singh and Manhas as well the recent survey article [11] of Singh and one of the authors.

A neighbourhood $G$ of 0 in $E$ is called shrinkable if $r \bar{G} \subseteq \operatorname{int} G$ for $0 \leq r<1$. By [5, Theorems 5 and 6], every Hausdorff topological vector space has a base of shrinkable neighbourhoods of 0 and also the Minkowski functional $\rho_{G}$ of any such neighbourhood $G$ is continuous. For details, we refer to $[2,3]$.

For any $t \in E$ and for $f \in C(X)$, the function $f_{t}$ defined by setting $f_{t}(x)=f(x) t$ for all $x \in X$ clearly belongs to $C(X, E)$. In particular, the constant $t$-function $1_{t}$ belongs to $C(X, E)$. The conditions under which $1_{t}$ belongs to $C V_{a}(X, E)$ are recorded in the following proposition: 
Proposition 1. Let $\mathcal{N}$ be a base of neighbourhoods of 0 in $E$. Then the following statements are equivalent:

(1.a) Every $v \in V$ is bounded (respectively, vanishes at infinity) on $X$.

(1.b) For every $t \in E, 1_{t} \in C V_{b}(X, E)$ (respectively, $C V_{0}(X, E)$ )

(1.c) Every constant selfmap on $X$ induces a composition operator on $C V_{b}(X, E)$ (respectively, $\left.C V_{0}(X, E)\right)$.

Proof. We may assume that $\mathcal{N}$ consists of closed, balanced and shrinkable sets. The proof then follows from Propositions 2.1. and 2.2 of [13] by replacing the continuous seminorms $p$ and $q$ respectively by the Minkowski functionals $\rho_{G}$ and $\rho_{H}$ of shrinkable neighbourhoods $G$ and $H$ of 0 in $E$.

\section{Characterization of WCOs}

In this section, we present necessary and sufficient conditions for $W_{\pi, T}$ to be a WCO on the weighted spaces $C V_{b}(X, E)$ and $C V_{0}(X, E)$. To avoid trival cases, we assume that for every $x \in X$, there exists an $h_{x} \in C V_{0}(X)$ such that $h_{x}(x) \neq 0$. This holds, in particular, when $X$ is locally compact or when each $v \in V$ vanishes at infinity on $X$.

In the locally convex setting, a characterization of $\mathrm{WCO}$ on $C V_{b}(X, E)$ has been presented by Singh and one of the authors in [10] under the assumption that $\pi(X)$ is equicontinuous whereas on $C V_{0}(X, E)$ it has been reported by Manhas in [6] but under the condition that $X$ is a $k_{\mathbb{R}}$-space. For non-locally convex spaces, multiplication operators on weighted spaces have been studied by Khan and Thaheem [4] with the same requirement as in [6]. It can be noted that either of the condition that " $\pi(X)$ is equicontinuous" or " $X$ is a $k_{\mathbb{R}}$-space" is needed only to make the map $M_{\pi}$ continuous (cf. [15]). Also, the results in $[4,6,10,14]$ characterizing WCOs and multiplication operators on weighted spaces require as a hypothesis that $T$ is continuous on whole of $X$ and $\pi$ is continuous in the uniform operator topology. But for instance when $\pi(x)=0$ for all $x \in X, W_{\pi, T}$ is a continuous operator even if $T$ is not continuous on whole of $X$. We now see that $\pi$ is continuous in the strong operator topology as follows:

If the system $V$ of weights satisfies condition (1.a) of Proposition 1 above, and $W_{\pi, T}$ is a $\mathrm{WCO}$ on $C V_{a}(X, E)$, where $a \in\{b, 0\}$, then, for any $t \in E, W_{\pi, T} 1_{t} \in C V_{a}(X, E)$ and $W_{\pi, T} 1_{t}(x)=\pi(x) t$ for all $x \in X$. Further, if $\left\{x_{\alpha}\right\}$ is a net in $X$ such that $x_{\alpha} \rightarrow x$ in $X$, then for every shrinkable neighbourhood $G$ of 0 in $E$, we have

$$
\rho_{G}\left[\pi\left(x_{\alpha}\right)-\pi(x)\right](t)=\rho_{G}\left[W_{\pi, T} 1_{t}\left(x_{\alpha}\right)-W_{\pi, T} 1_{t}(x)\right] \rightarrow 0
$$

This implies that $\pi \in C\left(X, B_{s}(E)\right)$. Note that $\pi$ is not necessarily continuous in the uniform operator topology (see [1] for example).

Theorem 2. Let $\mathcal{N}$ be a base of neighbourhoods of 0 in $E, T: X \rightarrow X$ and $\pi: X \rightarrow$ $B(E)$ such that $\pi(x) \neq 0$ for all $x \in X$. Assume that $\pi(X)$ is equicontinuous (or $X$ is $a k_{\mathbb{R}}$-space). Then the following conditions are sufficient for the pair $(\pi, T)$ to induce a $W C O$ on $C V_{b}(X, E)$ : 
(2.1) $\pi \in C\left(X, B_{s}(E)\right)$;

(2.2) $T$ is continuous;

(2.3) for every $v \in V$ and $G \in \mathcal{N}$, there eixsts $u \in V$ and $H \in \mathcal{N}$ such that

$$
v(x) \rho_{G}(\pi(x) t) \leq u o T(x) \rho_{H}(t) \text { for all } x \in X \text { and } t \in E .
$$

Furthermore, if the system $V$ consists of bounded weights on $X$, then the above conditions (2.1)-(2.3) are also necessary for $(\pi, T)$ to induce a WCO on $C V_{b}(X, E)$.

Proof. We may assume that $\mathcal{N}$ consists of closed, balanced and shrinkable sets.

Sufficient Part. Assume that (2.1)-(2.3) hold. It can be shown that $W_{\pi, T}$ take $C V_{b}(X, E)$ into itself as proved in [10] using [14, 15] with the corresponding seminorms replaced by the Minkowski functionals. To see the continuity of $W_{\pi, T}$, let $\left\{f_{\alpha}: \alpha \in \Delta\right\}$ be a net in $C V_{b}(X, E)$ such that $f_{\alpha} \rightarrow 0$, and let $v \in V$ and $G \in \mathcal{N}$. Using (2.3), choose $u \in V$ and $H \in \mathcal{N}$ such that $v(x) \rho_{G}(\pi(x) t) \leq u\left(T(x) \rho_{H}(t)\right.$ for all $x \in X$ and $t \in E$. Since $f_{\alpha} \rightarrow 0$, their exists an $\alpha_{0} \in \Delta$ such that $f_{\alpha} \in N(u, H)$ for all $\alpha \geq \alpha_{0}$. Then, for any $x \in X$ and $\alpha \geq \alpha_{0}$, we have

$$
v(x) \rho_{G}\left(\pi(x)\left(f_{\alpha} o T(x)\right)\right) \leq u o T(x) \rho_{H}\left(f_{\alpha} o T(x)\right)<1,
$$

or equivalently, $v(x) W_{\pi, T} f_{\alpha}(x) \in G$. Thus $w_{\pi, T}$ is continuous at 0 and hence, by its linearity, on $C V_{b}(X, E)$.

Necessary Part. Assume that all weights in $V$ are bounded. Then, as noted above, (2.1) holds while the proof of (2.2) is similar the one given in Theorem 2.2 of [12]. To prove (2.3), let $v \in V$ and $G \in \mathcal{N}$. Then, by continuity of $W_{\pi, T}$, there exists $u \in V$ and $H \in \mathcal{N}$ such that

$$
W_{\pi, T}(N(u, H)) \subseteq N(v, G) .
$$

We claim that $v(x) \rho_{G}(\pi(x) t) \leq 3 u o T(x) \rho_{H}(t)$ for all $x \in X$ and $t \in E$. To prove this, let $x_{0} \in X$ and $s \in E$ be fixed, take $y=T\left(x_{0}\right)$, and set $\varepsilon=u(y) \rho_{H}(s)$. Then consider the following cases:

$$
\begin{aligned}
& \text { (I) } \quad \varepsilon \neq 0 ; \quad \text { (II) } \varepsilon=0 \text {; that is, } \quad \text { (IIa) } u(y)=0, \quad \rho_{h}(s) \neq 0 \text {; } \\
& \text { (IIb) } u(y) \neq 0, \rho_{H}(s)=0 ; \quad \text { (IIc) } u(y)=0, \rho_{H}(s)=0 \text {. }
\end{aligned}
$$

Case (I): Suppose $\varepsilon \neq 0$. Then the set $D_{1}=\left\{x \in X: u(x) \rho_{H}(s)<3 \varepsilon\right\}$ is an open neighbourhood of $y$ and so according to Nachbin's Lemma [7, page 69], there exists an $f \in$ $C V_{b}(X)$ such that $0 \leq f \leq 1, f(y)=1$, and $f\left(X \backslash D_{1}\right)=\{0\}$. Write $h=(1 / 3 \varepsilon) f_{s}$. Then $h \in C V_{b}(X, E)$ and for any $x \in X, \rho_{H}(u(x) h(x))=(1 / 3 \varepsilon) u(x) f(x) \rho_{H}(s) \leq 1$, which means that $h \in N(u, H)$. By $(*), W_{\pi, T} h \in N(v, G)$ which gives $v(x) \rho_{G}(\pi(x) h o T(x)) \leq 1$ for every $x \in X$. This inequality when evaluated at $x_{0}$ gives $v\left(x_{0}\right) \rho_{G}\left(\pi\left(x_{0}\right) s\right) \leq 3 \varepsilon=$ $3 u o T\left(x_{0}\right) \rho_{H}(s)$. 
Case (IIa): When $u(y)=0, \rho_{H}(s) \neq 0$, we argue by contradiction. Suppose $v\left(x_{0}\right) \rho_{G}\left(\pi\left(x_{0}\right) s\right)>0$ and write $\delta=(1 / 3) v\left(x_{0}\right) \rho_{G}\left(\pi\left(x_{0}\right) s\right)$. Then the set $D_{2}=\{x \in$ $\left.X: u(x)<\delta\left[\rho_{H}(s)\right]^{-1}\right\}$ is an open neighbourhood of $y$ and so again by Nachbin's lemma there exists an $f \in C V_{b}(X)$ such that $0 \leq f \leq 1, f(y)=1$ and $f\left(X \backslash D_{2}\right)=$ $\{0\}$. Write $h=(1 / \delta) f_{s}$. Then $h \in N(u, H)$ and $W_{\pi, T} h \in N(v, G)$, which implies that $v(x) \rho_{G}(\pi(x) h o T(x)) \leq 1$ for every $x \in X$. This when evaluated at $x=x_{0}$ gives $u\left(x_{0}\right) \rho_{G}\left(\pi\left(x_{0}\right) s\right) \leq \delta$, which is absurd. Thus we must have $v\left(x_{0}\right) \rho G\left(\pi\left(x_{0}\right) s\right)=0$. For cases (IIb) and (IIc), we take $D_{3}=\{x \in X: u(x)<\delta+u(y)\}$ and $D_{4}=\{x \in X: u(x)<$ $\delta\}$ respectively as open neighbourhood of $y$ and proceed in the same way as in case (IIa). Thus, for all cases, we have $v\left(x_{0}\right) \rho_{G}\left(\pi\left(x_{0}\right) s\right) \leq u o T\left(x_{0}\right) \rho_{H}(s)$. Since $x_{0} \in X$ and $s \in E$ are arbitrarily taken, we conclude that $v(x) \rho_{G}(\pi(x) t) \leq 3 u o T(x) \rho_{H}(t)$ for all $x \in X$ and $t \in E$, proving our claim as well as the proof of the theroem.

The conditions of Theorem 2 above are not sufficient for the pair $(\pi, T)$ to induce a WCO on $C V_{0}(X, E)$ as already noted in [17, page 307] for the scalar valued space $C V_{0}(X)$. In the following theorem, we shall present a necessary and sufficient condition for $W_{\pi, T}$ to be a WCO on $C V_{0}(X, E)$, which also answers a remark given in [16, page 145] in the present setting.

Theorem 3. Let $\mathcal{N}$ be a base of neighbourhoods of 0 in $E, T: X \rightarrow X$ and $\pi: X \rightarrow$ $B(E)$ such that $\pi(x) \neq 0$ for all $x \in X$. Assume that $\pi(X)$ is equicontinuous (or $X$ is a $k_{\mathbb{R}}$-space). Then the following conditions are sufficient for the pair $(\pi, T)$ to induce a $W C O$ on $C V_{0}(X, E)$ :

(3.1) $\pi \in C\left(X, B_{s}(E)\right)$;

(3.2) $T$ is continuous;

(3.3) for every $v \in V$ and $G \in \mathcal{N}$, there exists $u \in V$ and $H \in \mathcal{N}$ such that $v(x) \rho_{G}(\pi(x) t)$ $\leq u o T(x) \rho_{H}(t)$ for all $x \in X$ and $t \in E$.

(3.4) for every $v \in V, G \in \mathcal{N}, \varepsilon>0$ and compact subset $K$ of $X$, the set $T^{-1}(K) \cap K_{t}$ is compact for all $t \in E$, where $X_{t}=\left\{x \in X: v(x) \rho_{G}(\pi(x) t) \geq \varepsilon\right\}$.

Furthermore, the above conditions (3.1)-(3.4) are necessary for $(\pi, T)$ to induce a WCO on $C V_{0}(X, E)$ if all the weights in $V$ vanish at infinity on $X$.

Proof. We may assume that $\mathcal{N}$ consists of closed, balanced and shrinkable sets.

Sufficient Part. Assume that (3.1)-(3.4) hold. Then, by Theorem 2 above, $W_{\pi, T}$ is a WCO on $C V_{b}(X, E)$. We show that $C V_{0}(X, E)$ is invariant under $W_{\pi, T}$. For this, let $f \in C V_{0}(X, E), v \in V$ and $G \in \mathcal{N}$. By (3.3) choose $u \in V$ and $H \in \mathcal{N}$ such that

$$
v(x) \rho_{G}(\pi(x) t) \leq u o T(x) \rho_{H}(t) \text { for all } x \in X \text { and } t \in E .
$$

Since $f \in C V_{0}(X, E)$, given any $\varepsilon>0$ there exists a compact subset $K$ of $X$ such that $u(x) \rho_{H}(f(x))<\varepsilon$ for all $x \in X \backslash K$. Also, for any $t \in E$, set $K_{t}=T^{-1}(K) \cap X_{t}$ is compact, in view of (3.4). Now if $x \notin K_{t}$, then either $x \notin T^{-1}(K)$ or $x \notin X_{t}$. First suppose that $x \notin T^{-1}(K)$. Then $T(x) \in X \backslash K$, and so we have $u o T(x) \rho_{H}(f o T(x))<\varepsilon$. By $(* *)$, this implies that $v(x) \rho_{G}(\pi(x)(f o T(x)))<\varepsilon$. If $x \notin X_{t}$, then $v(x) \rho_{G}(\pi(x) t)<\varepsilon$ 
and in particular this implies that $v(x) \rho_{G}(\pi(x)(f o T(x)))<\varepsilon$. Thus $v W_{\pi, T} f: X \rightarrow E$ vanishes at infinity. Since $v \in V$ was arbitrary, we conclude that $W_{\pi, T} f \in C V_{0}(X, E)$.

Necessary Part. Assume that all the weights in $V$ vanish at infinity and let $W_{\pi, T}$ be a WCO on $C V_{0}(X, E)$. Then the conditions (3.1)-(3.3) can be proved in a similar way as in Theorem 2 above. To prove (3.4), take $v \in V, G \in \mathcal{N}, \varepsilon>0$ and let $K$ be a compact subset of $X$. Then by Nachbin's Lemma [7, page 69] there exists an $f \in C V_{0}(X)$ such that $0 \leq f \leq 1$ and $f(K)=\{1\}$. For any $t \in E$, the function $f_{t} \in C V_{0}(X, E)$ since weights are vanishing. So $W_{\pi, T} f_{t} \in C V_{0}(X, E)$, which means that the set $A=\{x \in X: v(x) \rho G(\pi(x)(f o T(x))) \geq \varepsilon\}$ is compact. We note that $K_{t}=T^{-1}(K) \cap X_{t}$ is a closed subset of $A$. Hence $K_{t}$ is compact.

Remarks. (i) Theorems 2 and 3 above improve Theorem 3.1 of [4], Theorem 2.1 of [6], Theorem 3.2 of [10], Theorem 2.1 of [14] and Theorems 3.3 and 3.4 of [9] as well as extend Theorem 2.2 of [12] to a non-locally convex setting.

(ii) On non-locally convex weighted spaces $F V_{b}(X, E)$ and $F V_{0}(X, E)$ of $E$-valued functions (not necessarily continuous) on a topological space $X$, weighted composition operators have been studied by two of the authors in [8].

\section{References}

[1] J. E. Jamison and M. Rajagopalan, Weighted Composition Operator on $C(X, E)$, J. Operator Theory, 19(1988), 307-317.

[2] L. A. Khan, Weighted topology in the non-locally convex setting, Mat. Vesnik, 27(1985), 189-195.

[3] L. A. Khan and A. B. Thaheem, Multiplication operators on weighted spaces in the nonlocally convex frame work, Internat. J. Math and Math. Sci., 20(1977), 75-80.

[4] L. A. Khan and A. B. Thaheem, Operator-valued Multiplication operators on weighted function spaces, preprint.

[5] V. Klee, Shrinkable neighbourhoods in Hausdorff linear spaces, Math. Ann., 141(1960), 281-285.

[6] J. S. Manhas, Weighted composition operators on weighted spaces of continuous functions, Contemporary Mathematics, 213(1998), 99-119.

[7] L. Nachbin, Elements of approximation theory, Math. Studies 14, Van Nostrand, Princeton, 1967.

[8] Bhopinder Singh and Kamaljeet Kour, On weighted composition operators on non-locally convex function spaces, Indian Journal of Pure and Applied Math., 28(1997), 1505-1512.

[9] Bhopinder Singh and Kamaljeet Kour, WCOs on non-locally convex weighted spaces of continuous functions, J. Indian Math. Soc., 66(1999).

[10] Bhopinder Singh and R. K. Singh, A characterization of weighted composition operators, Rocky Mountain J. Math, 23(1993), 1107-1114.

[11] Bhopinder Singh and R. K. Singh, Compact weighted composition operators on spaces of continuous functions: A survey, Extracta Math., 10(1995), 1-20.

[12] Bhopinder Singh and R. K. Singh, Weighted composition operators and dynamical systems, Tamkang J. Math., 29(1998), 101-107. 
[13] Bhopinder Singh, R. K. Singh and J. S. Manhas, Compact operators of composition on some locally convex function spaces, J. Operator Theory, 31(1994), 11-20.

[14] R. K. Singh and J. S. Manhas, Multiplication operators and dynamical systems, J. Austral. Math. Soc., (Series A), 53(1992), 92-102.

[15] R. K. Singh and J. S. Manhas, Corrigendum to Multiplication operators and dynamical systems, J. Austral Math. Soc. (Series A), 58(1995), 142.

[16] R. K. Singh and J. S. Manhas, Composition operators on function spaces, North-Holland Math. Studies 179, Amsterdam, 1993.

[17] R. K. Singh and W. H. Summers, Composition operators on weighted spaces of continuous functions, J. Austral. Math. Soc. (Series A), 45(1988), 303-319.

Department of Mathematics, University of Jammu, Jammu-180 004, India.

Department of Mathematics, Govt. College of Engineering and Technology, Canal Road, Jammu-180 001, India. 